\title{
Searches for New Physics in ep Collisions
}

\author{
Uwe Schneekloth
}

DESY

Lake Louise Winter Institute

23.02.2007 


\section{Searches for New Physics at HERA}

Recent results

Model dependent searches

- Leptoquarks

- Lepton flavor violation

- Excited fermions

- Single top production

- Double charged Higgs

- Supersymmetry

Limits from precision measurements

- NC DIS: contact interactions, extra large dimensions, quark radius

- CC DIS: right-handed weak currents
Model independent searches

- Events with isolated leptons and missing $\mathrm{P}_{\mathrm{T}}$

- General searches

- Tau production

- Multi-lepton production

- Magnetic monopoles 


\section{The HERA ep Collider at DESY}
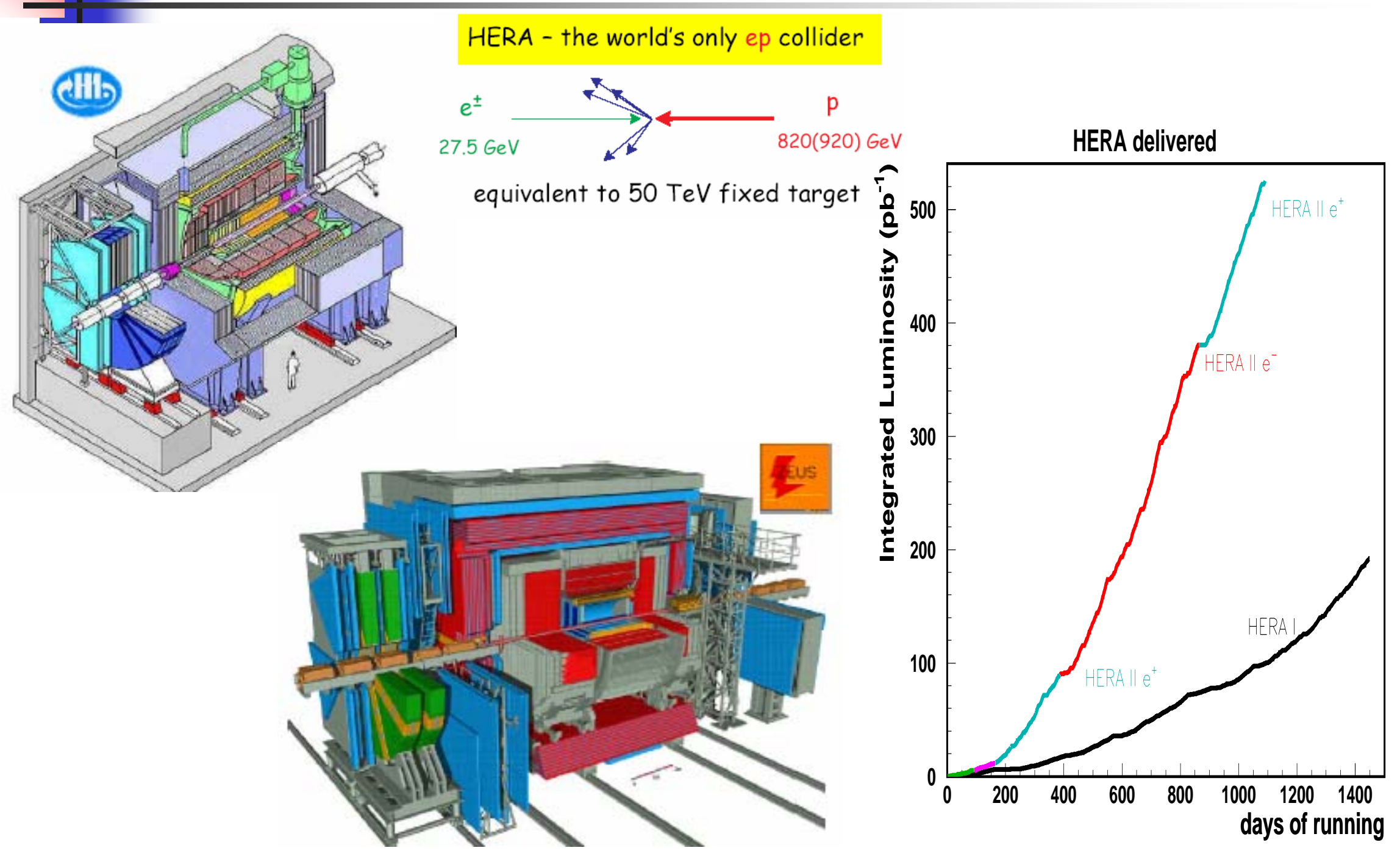

Searches at HERA

U. Schneekloth 


\section{Isolated Leptons and $\mathrm{p}_{T}{ }^{\text {miss }}$}
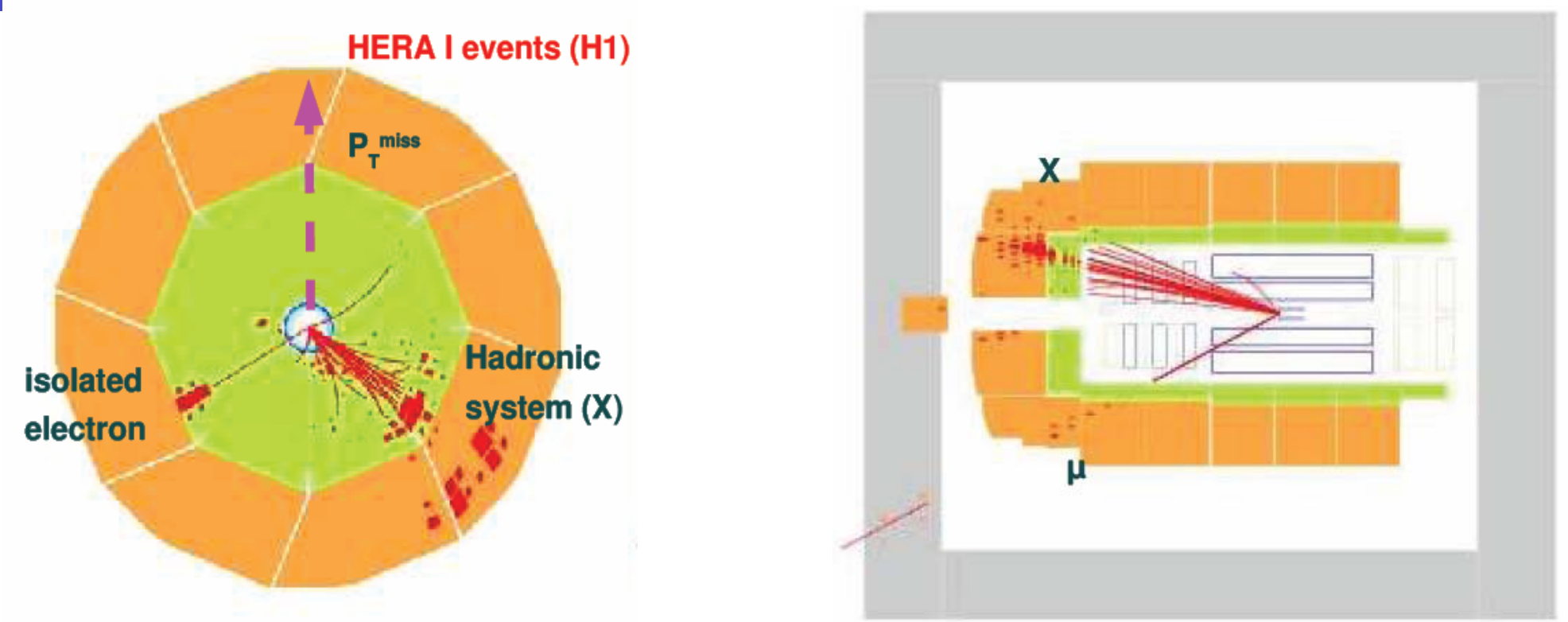

Excess of events with isolated leptons and large missing transverse momentum observed by $\mathrm{H} 1$ since 1993 , mainly in $\mathrm{e}^{+} \mathrm{p}$ collisions.

- HERA I data: $\mathrm{p}_{T}{ }^{\text {miss }}>25 \mathrm{GeV} 11$ events/3.5 \pm 0.6 (standard model)

- Excess not observed by ZEUS

- Now almost final (HERA II) data set available.

- Common working group, detailed comparisons. 


\section{Isolated Leptons and $\mathrm{p}_{T}{ }^{\text {miss }}$}

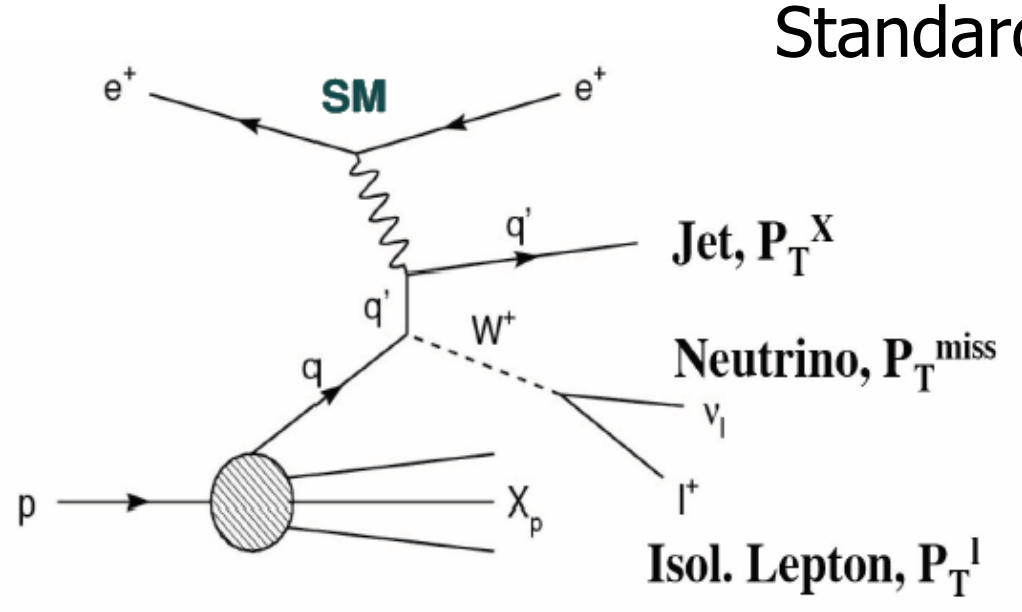

- At HERA $\sigma \sim 1.2 \mathrm{pb}$

- Important background to physics beyond the standard model

Event selection

- $\mathrm{P}_{\mathrm{T}}{ }^{\prime}>10 \mathrm{GeV}, \mathrm{P}_{\mathrm{T}}$ miss $>12 \mathrm{GeV}$

- Lepton isolation

- Event balance (acoplanarity)

- Kinematic and topological variables

- H1 and ZEUS slightly different $\theta$ ranges 


\section{Isolated Leptons and $\mathrm{p}_{T}^{\text {miss }}$}

$\mathrm{H} 1$ results

$$
\text { e-p } 184 \mathrm{pb}^{-1}
$$

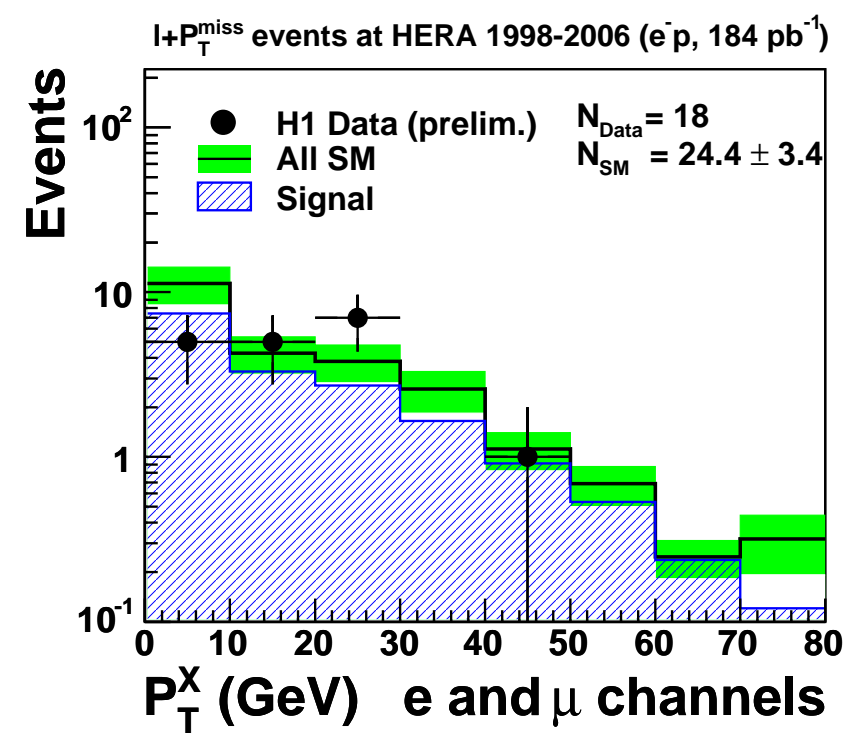

No excess in e-p data

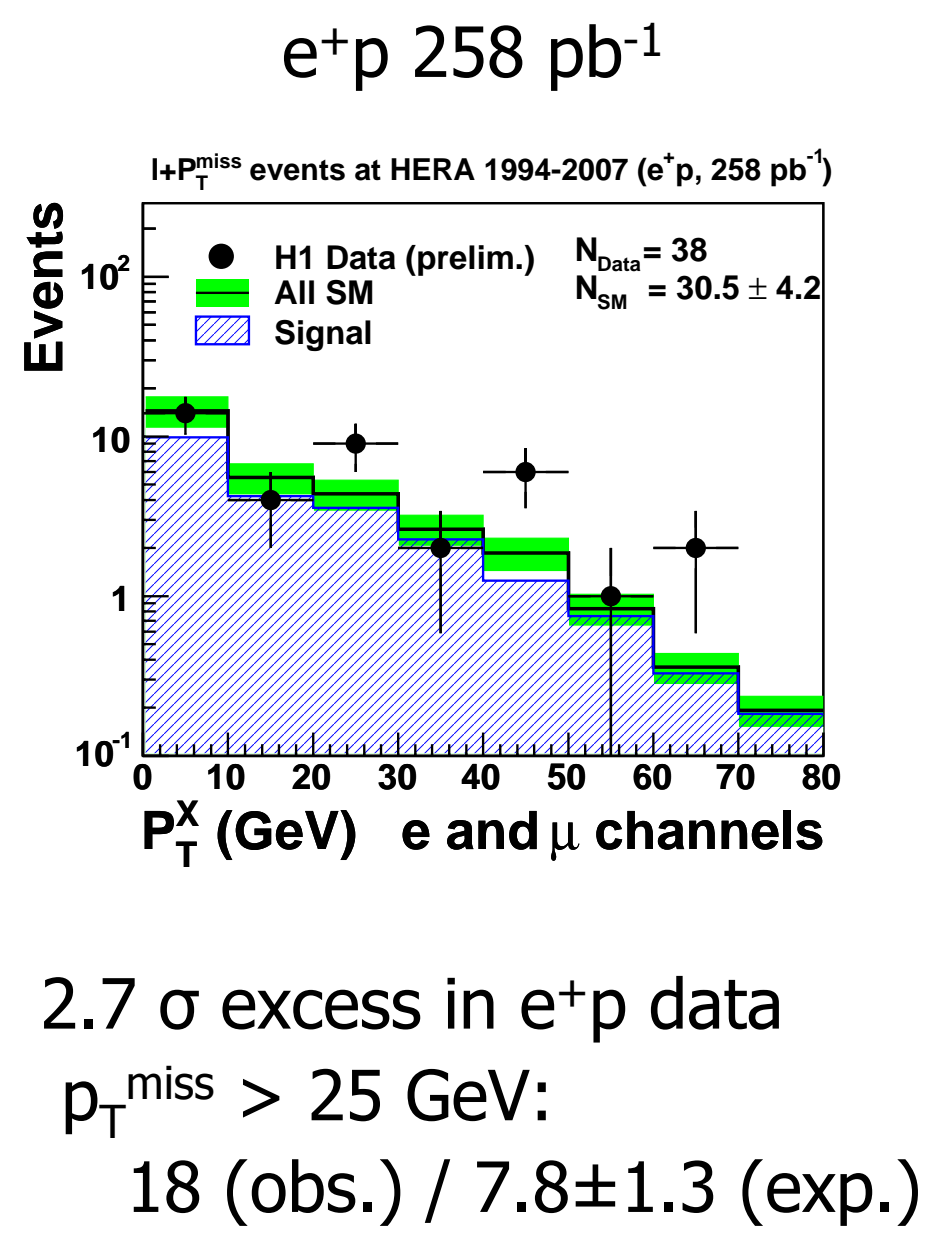




\section{Isolated Leptons and $\mathrm{p}_{T}{ }^{\text {miss }}$}

Comparison of $\mathrm{H} 1$ and ZEUS results (preliminary)

\begin{tabular}{|l|l|l|l|}
\hline $\mathrm{p}_{\mathrm{T}}{ }^{\mathrm{x}}>25 \mathrm{GeV}$ & $\begin{array}{l}\text { electron } \\
\text { obs./exp. }\end{array}$ & $\begin{array}{l}\text { muon } \\
\text { obs./exp }\end{array}$ & $\begin{array}{l}\text { Combined } \\
\text { obs./exp }\end{array}$ \\
\hline $\begin{array}{l}\mathrm{H} 1 \text { e p } \\
1998-2006184 \mathrm{pb}^{-1}\end{array}$ & $3 / 3.8 \pm 0.6(61 \%)$ & $0 / 3.1 \pm 0.5(74 \%)$ & $3 / 6.9 \pm 1.0$ \\
\hline $\begin{array}{l}\mathrm{H} 1 \text { e } \\
1994-2007258 \mathrm{pb}^{-1}\end{array}$ & $10 / 4.1 \pm 0.8(75 \%)$ & $8 / 3.7 \pm 0.6 \mathrm{I}(85 \%)$ & $18 / 7.8 \pm 1.3$ \\
\hline $\begin{array}{l}\text { ZEUS e-p } \\
1998-2006204 \mathrm{pb}^{-1}\end{array}$ & $5 / 3.8 \pm 0.6(55 \%)$ & $2 / 2.2 \pm 0.3(86 \%)$ & $7 / 6.0 \pm 0.7$ \\
\hline $\begin{array}{l}\text { ZEUS e+p } \\
1996-2006228 \mathrm{pb}^{-1}\end{array}$ & $1 / 3.2 \pm 0.4(75 \%)$ & $3 / 3.1 \pm 0.5(80 \%)$ & $4 / 6.3 \pm 0.6$ \\
\hline
\end{tabular}

Standard model W photoproduction is given in parentheses

- ZEUS in good agreement with Standard Model W production

- H1 some excess at large $p_{T}{ }^{x}$ in $e^{+} p$ data $(2.7 \sigma)$.

Excess slightly less significant than HERA I and earlier HERA II data 


\section{Isolated Leptons and $\mathrm{p}_{T}^{\text {miss }}$}

\section{Comparison of efficiencies}

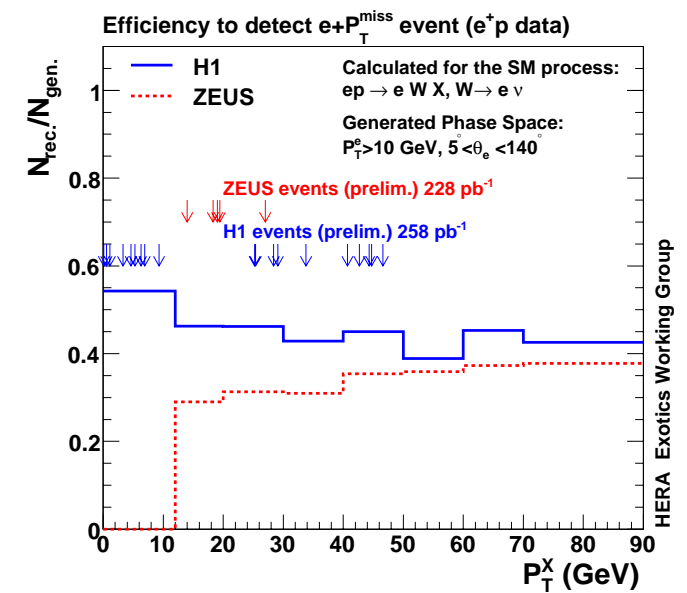

Efficiency to detect $e+P_{T}^{\text {miss }}$ events with $P_{T}^{x}>25 \mathrm{GeV}\left(e^{+} p\right)$

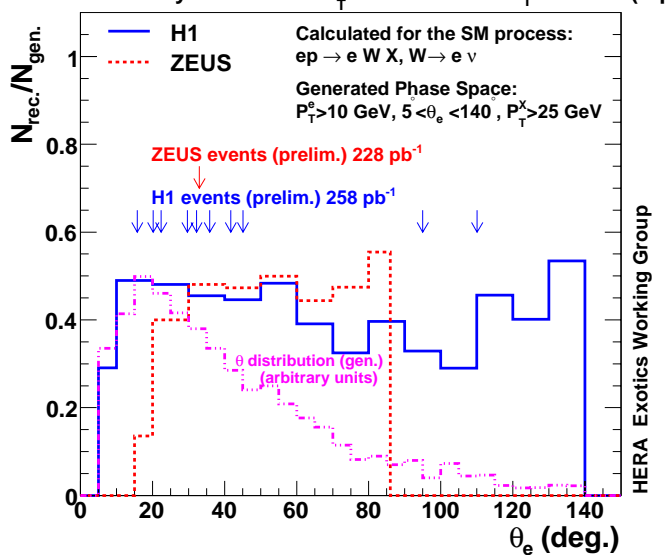

- Similar efficiencies

- ZEUS slightly less efficient due to different tracking requirements

Efficiency to detect $\mu+P_{T}^{\text {miss }}$ events ( $e^{+} p$ data)

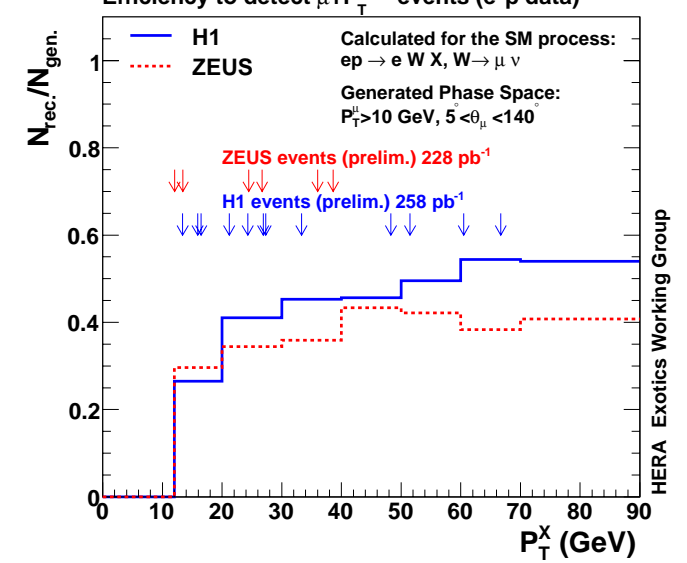

Efficiency to detect $\mu+P_{T}^{\text {miss }}$ events with $P_{T}^{x}>25 \mathrm{GeV}\left(e^{+} p\right)$

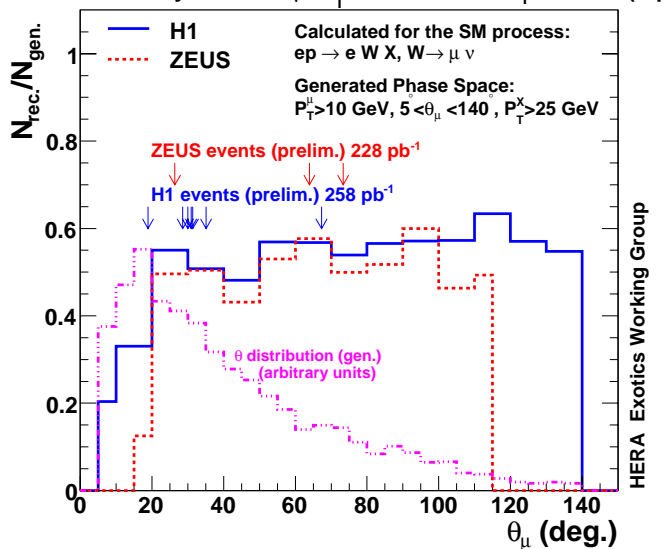

- Small difference for W photoproduction

- Small effect in high $p_{T}{ }^{x}$ region 


\section{General Search for High- $\mathrm{P}_{\mathrm{T}}$ Phenomena}

- H1 performed a model independent, generic search in final states with $\geq 2$ high $-\mathrm{P}_{\mathrm{T}}$ objects:

$$
\begin{aligned}
& \mathrm{e}, \mu, j e t s, \gamma, v \\
& \mathrm{P}_{\mathrm{T}}>20 \mathrm{GeV} \\
& 10^{\circ}<\theta<140^{\circ}
\end{aligned}
$$

- Classified by final state

- Standard model predictions for all HERA processes considered: NC and CC DIS, photoproduction, lepton-pairproduction, W-production, QEDC Good agreement of event yields with SM expectation for most classes
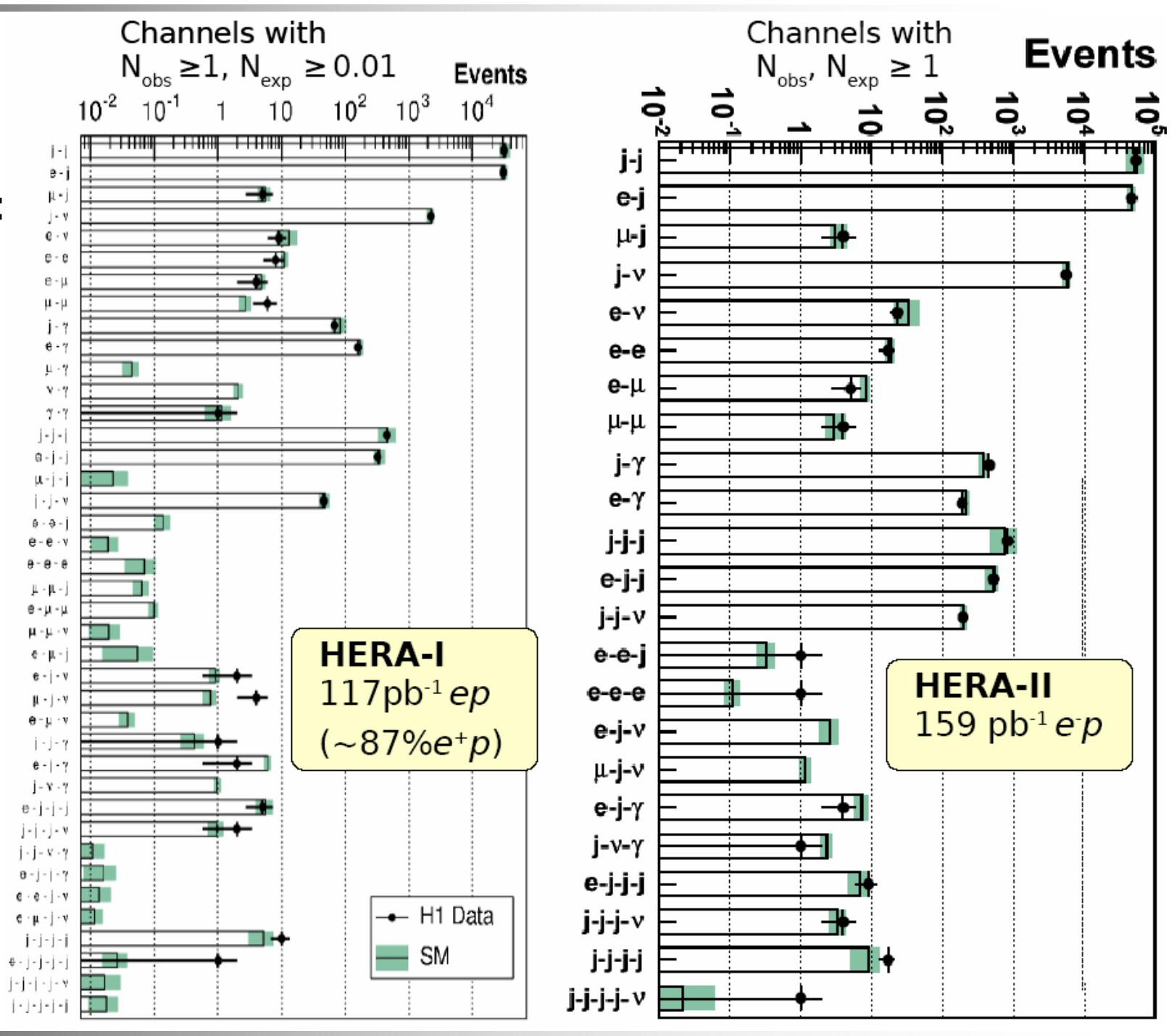

Searches at HERA

U. Schneekloth 


\section{Search for Excited Neutrinos}

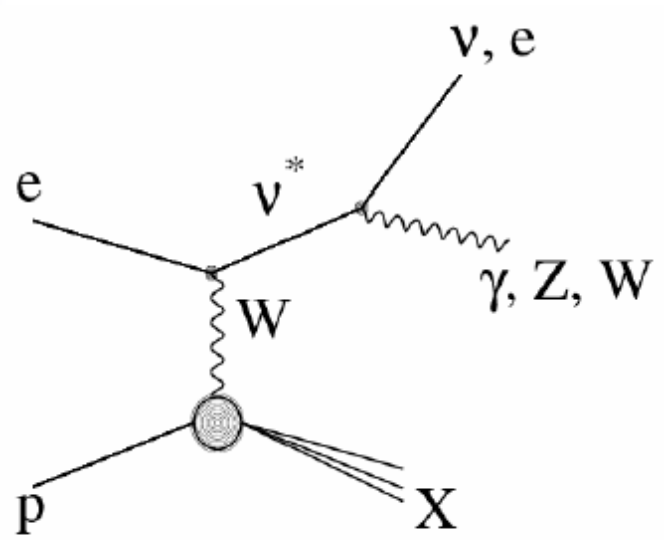

$v^{*}$ composite fermion at scale $\Lambda$

Cross section proportional to couplings $f$ and $f^{\prime}$ (electroweak) $\sigma\left(e^{-p}\right) \approx 100 \times \sigma\left(e^{+} p\right)$ Improved sensitivity with HERA II data
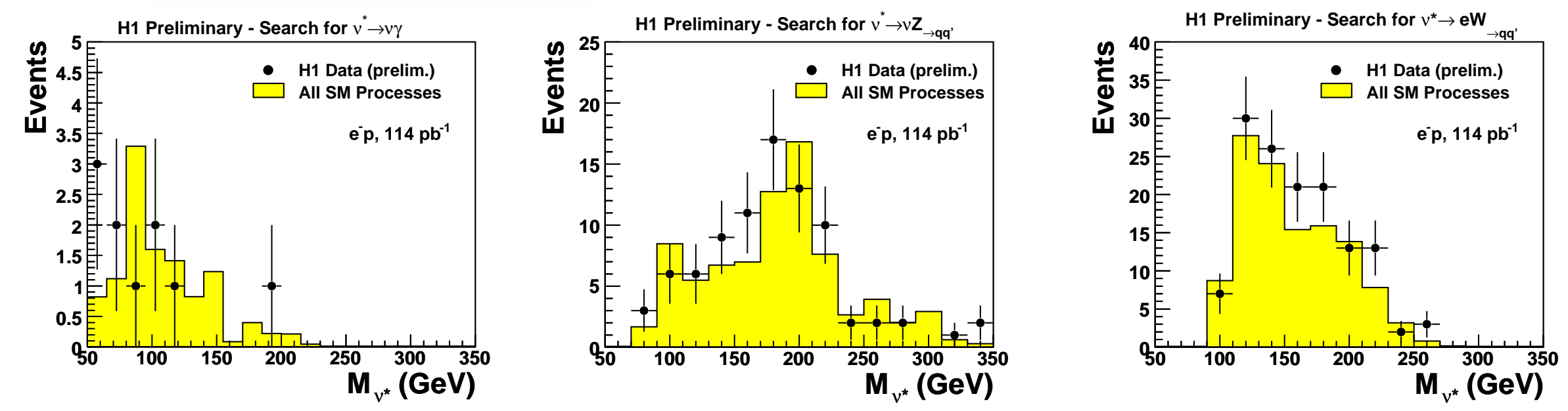

- Data well described by standard model predictions

- No evidence for excited neutrinos 


\section{Search for Excited Neutrinos}

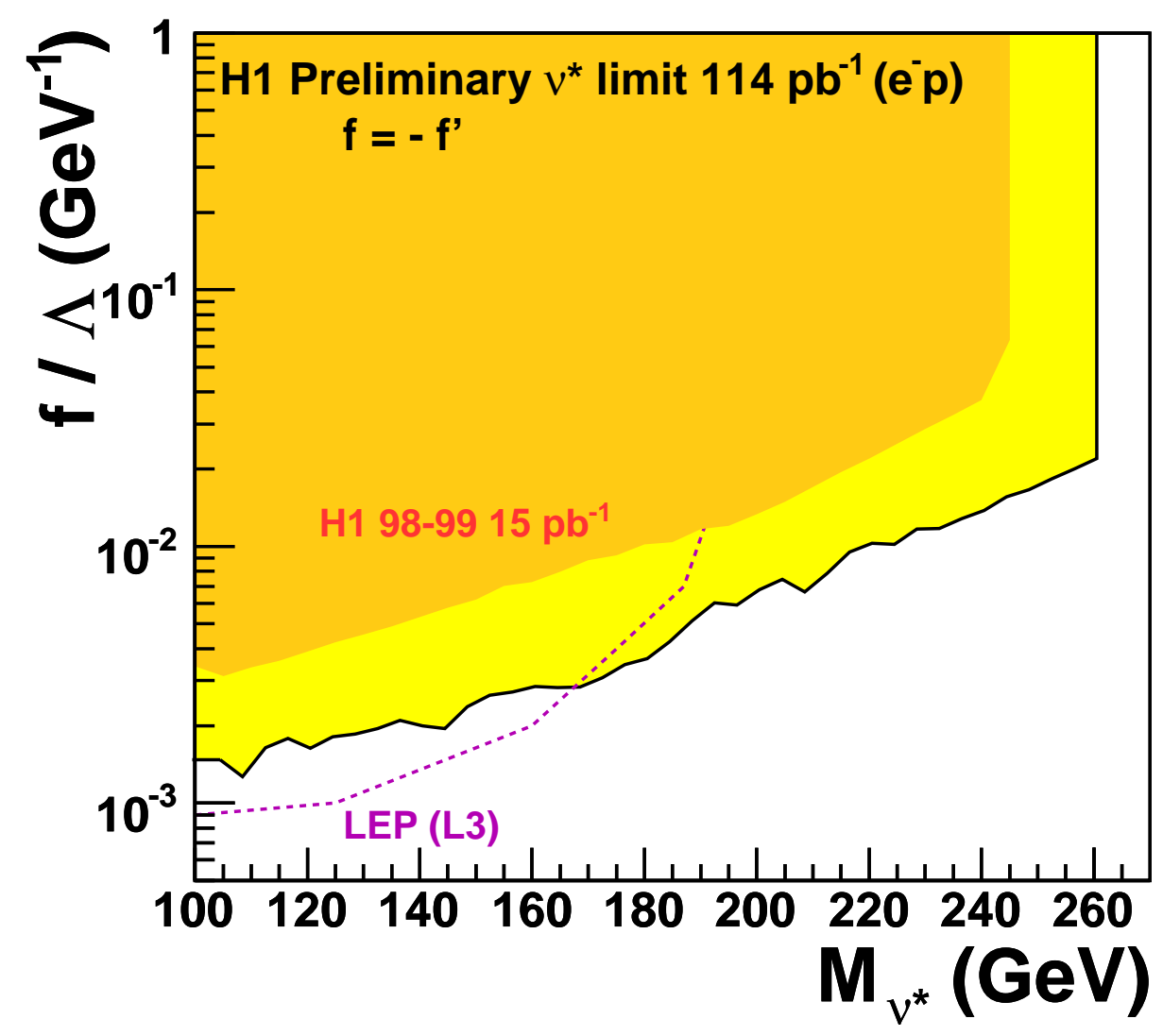

Exclusion limits for $f=-f^{\prime}$ (max. coupling to photon)

Similar limits for $f=+f^{\prime}$

Assuming $\mathrm{f} / \Lambda=1 / \mathrm{M}_{\mathrm{v}}$ : $M_{v}>188 \mathrm{GeV}$ at $95 \%$ C.L. 


\section{Contact Interactions}

- New interactions between electron and quarks involving mass scales above center-of-mass energy can modify cross section at high $\mathrm{Q}^{2}$ via virtual effects.

- Should observe deviations from Standard Model predictions.

- Many interactions, e.g. mediated by heavy leptoquarks, can be modeled as four-fermion contact interactions.

- Use SM predictions at high $\mathrm{Q}^{2}$ from evolution of accurate measurements of the proton structure functions made at lower $Q^{2}$ for searches for:

- four-fermion interactions,

- graviton exchange in models with extra large dimensions or

- finite charge radius of quarks (this talk). 


\section{Are Quarks Elementary?}

- Quark substructure can be detected by measuring spatial distribution of quark charge.

- If quark has finite radius, cross section will decrease as probe penetrates into it.

$$
\frac{d \sigma}{d Q^{2}}=\frac{d \sigma^{S M}}{d Q^{2}}\left(1-\frac{R_{q}^{2}}{6} Q^{2}\right) \quad \mathrm{R}_{\mathrm{q}} \text { is rms of electroweak charge }
$$

- Limit on quark size (assuming point-like electron)

- $\mathrm{R}_{\mathrm{q}}<0.8510^{-16} \mathrm{~cm}$

$(95 \% \mathrm{CL})$

- $\mathrm{R}_{\mathrm{q}}{ }^{2}<-\left(1.0610^{-16} \mathrm{~cm}\right)^{2}$

If charge changes sign as

function of radius

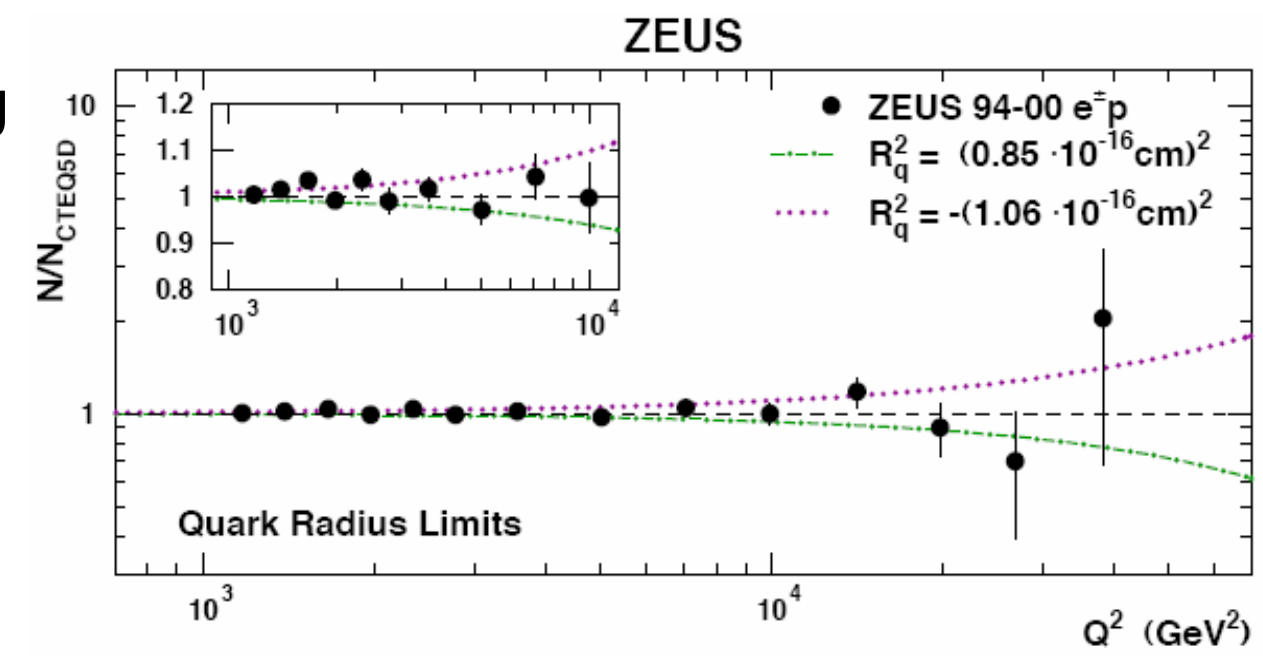

Similar limits by $\mathrm{H} 1, \mathrm{CDF}$ and $\mathrm{L} 3$. 


\section{Search for Lepton-Flavor Violation}

Motivation for search $\mathrm{e}^{+} \mathrm{p} \rightarrow \mu^{+}\left(\tau^{+}\right) \mathrm{X}$

- Many extensions of Standard Model allow lepton-flavor violation

- Lepton-flavor violation occurs in neutrino section as shown by neutrino oscillation experiments

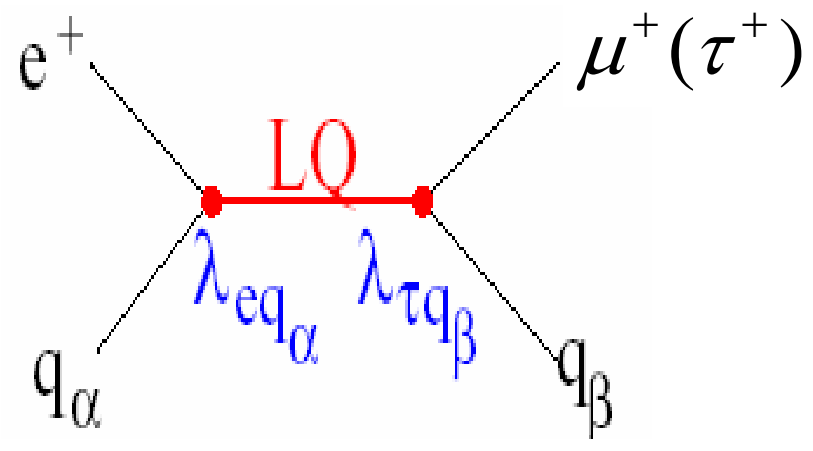

- Leptoquark (LQ): both lepton and baryon numbers and leptonquark Yukawa couplings

- LFV if LQ couples to two different generations

- Mediated by squarks in R-parity-violation SUSY models 


\section{Search for Leptoquarks}

Buchmüller-Rückl-Wyler model: 14 LQ types, fermionic number $(\mathrm{F}=3 \mathrm{~B}+\mathrm{L}) 0$ or 2

Narrow width approximation High mass approximation

$$
\begin{array}{cc}
\left(M_{L Q}<\sqrt{s}\right) \\
\sigma^{N W A}\left(\lambda, M_{L Q}\right) \propto \lambda_{l q_{i}}^{2} B_{l q_{j}}
\end{array}
$$

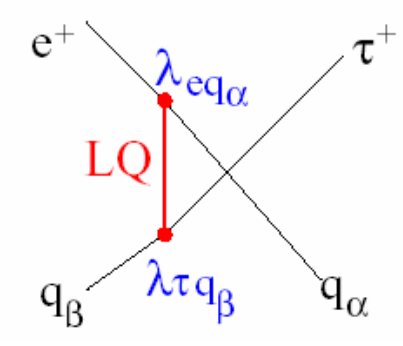




\section{Search for Leptoquarks}

Results: Limits on $\lambda_{e q_{1}}$ as function of $\mathrm{M}_{\mathrm{LQ}}$ for scalar and vector leptoquarks

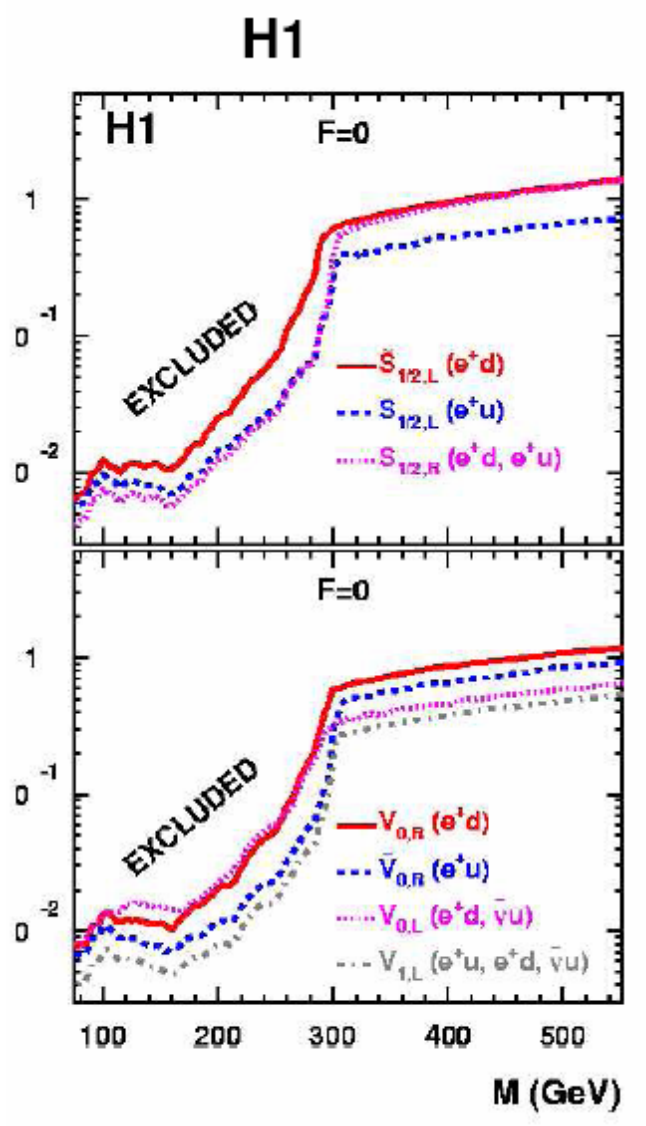

\section{ZEUS}

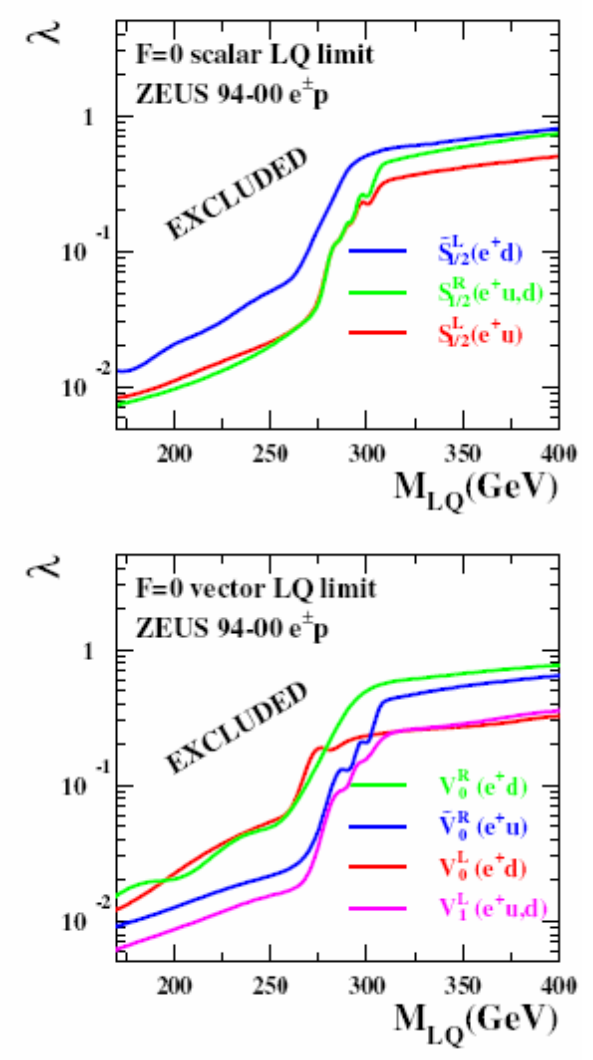

HERA I data

$\mathrm{F}=0$ scalar BRW LQ

Couples to eq only

$\mathrm{F}=0$ vector $\mathrm{BRW} L \mathrm{~L}$

eq and vq channels combined 


\section{Search for Leptoquarks}

Results: Limits on $\lambda_{e q_{1}}$ as function of $\mathrm{M}_{\mathrm{LQ}}$ for scalar and vector leptoquarks

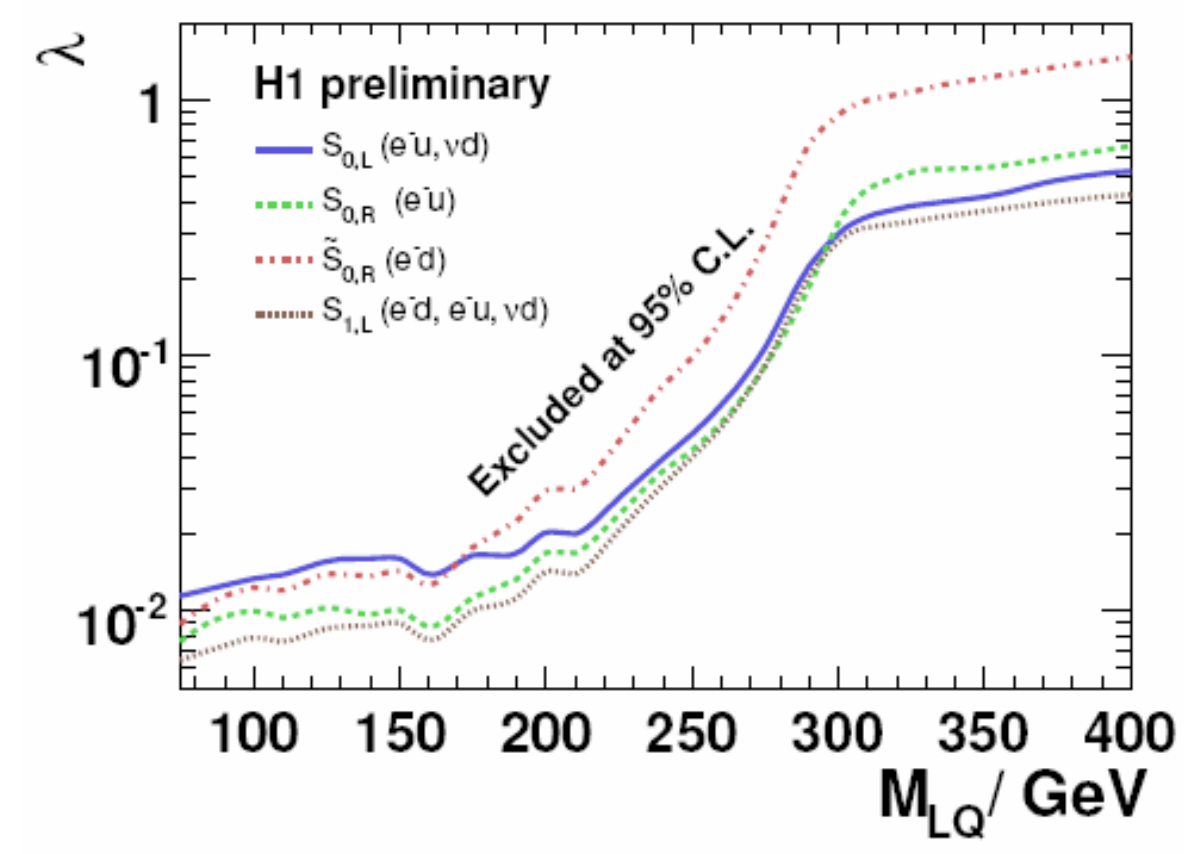

HERA II data

$\mathrm{F}=2$ scalar BRW LQ

e-p data more sensitive than $e^{+} p$

Similar limits for vector $L Q$

Assuming $\lambda=\sqrt{ } 4 \pi \alpha \approx 0.3$ lower limits on $\mathrm{M}_{\mathrm{LQ}} 276-304 \mathrm{GeV}$ 


\section{Conclusions}

\section{Searches for New Physics at HERA}

- Events with isolated leptons and missing $\mathrm{P}_{\mathrm{T}}$

- $\mathrm{H} 1$ still observing some excess at large $\mathrm{p}_{\mathrm{T}}{ }^{\mathrm{X}}$ in $\mathrm{e}^{+} \mathrm{p}$ data $(2.7 \sigma)$

- Slightly less significant than in HERA I and earlier HERA II data

- General search for high- $P_{T}$ phenomena

- Good agreement of event yields with standard model expectation for most classes of events

- Extended limits for mass of excited neutrinos with new e-p data

- Are quarks elementary?

- New limits on quark radius

- New limits on leptoquark masses 